\section{A case of severe posterior reversible encephalopathy syndrome in a preeclamptic woman in the early postpartum period}

\author{
Yasuhiro Miyoshi, Sachie Suga, Yoshinori Mizutani, \\ Masashi Fukuda, Hiroshi Yamashita, Ichiro Yasuhi \\ Department of Obstetrics and Gynecology, Nagasaki Medical Center, Nagasaki, Japan
}

Reprint request to:

Yasuhiro Miyoshi, MD., Department of Obstetrics and Gynecology, Nagasaki Medical Center, Omura, Nagasaki, 8568562, Japan.

E-mail: yasuhiro.miyoshi@gmail. com

\section{Key words:}

cerebral decompression, HELLP syndrome, posterior reversible encephalopathy syndrome (PRES), preeclampsia

Received: November 17, 2014

Revised: December 2, 2014

Accepted: December 12, 2014

DOI:10.14390/jsshp.2.94

Posterior reversible encephalopathy syndrome (PRES) presents with headaches, an altered mental status, seizures and visual loss, and generally exhibits a good prognosis. We herein report the case of a primiparous preeclamptic woman who developed severe PRES after cesarean delivery at term. She underwent emergency cesarean section due to the onset of HELLP syndrome at 37 weeks of gestation. She fell into a deep trance on the first day postpartum. Brain MRI showed diffuse brain edema involving the brainstem. Due to the considerable risk of brain herniation, we performed surgical decompression for brain swelling on that day in addition to intensive treatment for HELLP syndrome and DIC. The patient successfully recovered from the acute episode without any neurological deficits. Although most cases of PRES involve a good prognosis without the need for surgical intervention, physicians should keep in mind the potential for serious cases requiring decompressive brain surgery in women with PRES.

\section{Introduction}

Posterior reversible encephalopathy syndrome (PRES) is a reversible form of edema in the brain primarily involving the posterior lobe. ${ }^{1)}$ The clinical features of PRES include headaches, an altered mental status, seizures and visual loss. Neuroimaging is essential for confirming the diagnosis of PRES. ${ }^{2)}$ The onset of PRES is associated with preeclampsia/eclampsia, renal failure and the use of immunosuppressant drugs and steroids. ${ }^{1)}$ Although there have been a few cases with irreversible neurological complications or even maternal death, ${ }^{3)}$ the prognosis of PRES is generally considered to be good without neurological sequelae.

In this report, we present a serious case of PRES requiring decompressive brain surgery on the first day postpartum after cesarean delivery in a woman with preeclampsia and HELLP syndrome.

\section{Case Report}

A 31-year-old primigravida presented to our hospital at
37 weeks of gestation with a diagnosis of preeclampsia. Her medical history was unremarkable, and her pregnancy had remained uncomplicated until 34 weeks of gestation. At the time of a prenatal check-up at 36 weeks of gestation, she exhibited a blood pressure of 135/99 $\mathrm{mmHg}$ with $2+$ proteinuria. At 37 weeks of gestation, she was diagnosed as having preeclampsia with a blood pressure of $151 / 97 \mathrm{mmHg}$ and $2+$ proteinuria, and was referred to our hospital for treatment and hospitalization.

On admission, the patient had a blood pressure of $158 / 102 \mathrm{mmHg}$, with very mild bilateral lower extremity edema. She exhibited no vaginal bleeding, with cervical dilatation of $1.5 \mathrm{~cm}$. A nonstress test showed a reassuring fetal heart rate pattern, and her lab tests were unremarkable.

The next day, the patient suffered from a backache and dyspnea, with a blood pressure of 166/104 $\mathrm{mmHg}$ and the following laboratory test results: $\mathrm{AST}=83$ $\mathrm{IU} / 1, \mathrm{ALT}=53 \mathrm{IU} / 1, \mathrm{LDH}=315 \mathrm{IU} / 1, \mathrm{Hb}=13.3 \mathrm{~g} / \mathrm{dl}$, PLT $=151,000 / \mu 1$, and serum creatinine $=0.7 \mathrm{mg} / \mathrm{dl}$. We decided to perform emergency cesarean section due to the suspected development of early-stage HELLP 

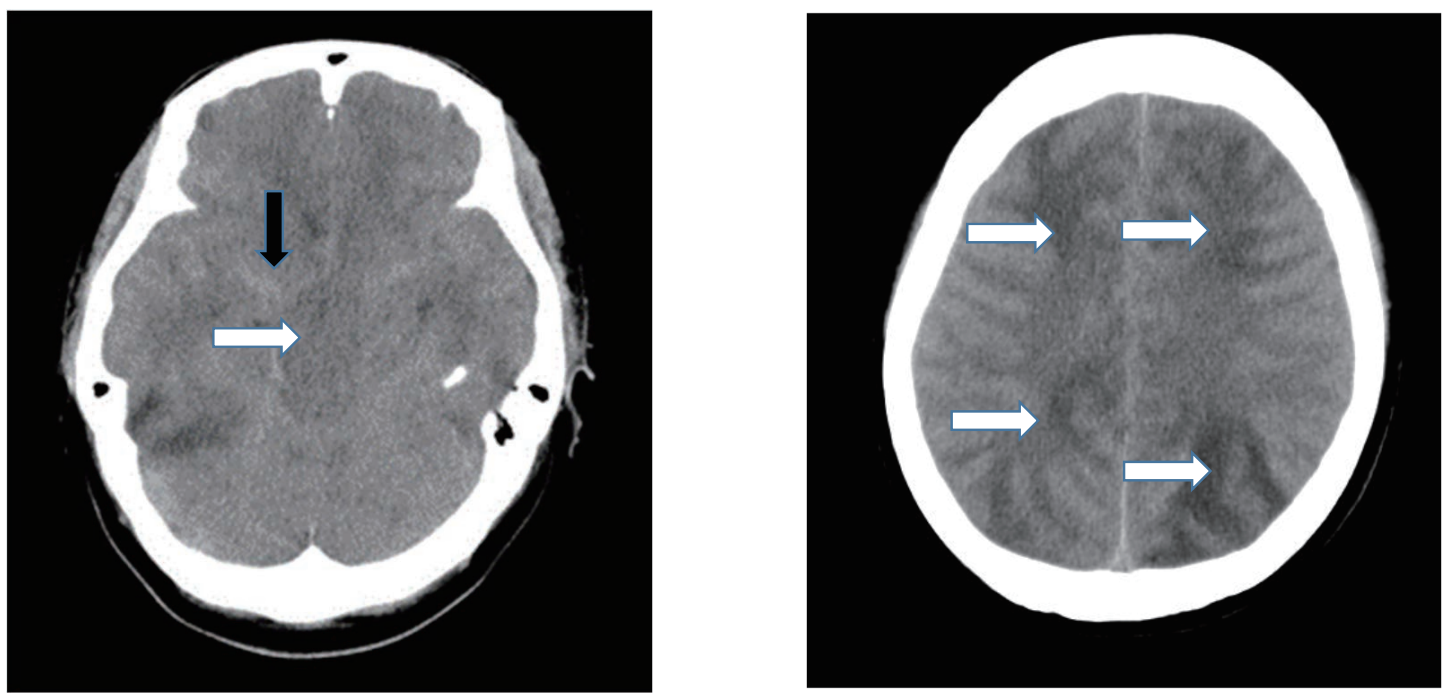

Figure 1. CT scan on postpartum day 1.

CT scan on postpartum day 1 shows slight high-density lesion in cerebral base (black arrow) and diffuse brain edema (white arrow) involving the brain stem.

syndrome. She delivered a male infant weighing $2,904 \mathrm{~g}$ with one- and five-minute Apgar scores of 8 and 9, respectively. Her general condition was stable during the cesarean section procedure under regional anesthesia, and the measured blood loss was $650 \mathrm{~g}$. Laboratory test results obtained immediately after the surgery were as follows: $\mathrm{AST}=203 \mathrm{IU} / 1, \mathrm{ALT}=114 \mathrm{IU} / 1, \mathrm{LDH}=497$ $\mathrm{IU} / 1, \mathrm{Hb}=13.3 \mathrm{~g} / \mathrm{dl}$, and PLT $=91,000 / \mu \mathrm{l}$. Treatment with intravenous magnesium sulphate was started after the cesarean section in order to prevent eclampsia. Intravenous nicardipine was administered to control hypertension.

On postpartum day 1, 26 hours after the cesarean section, and shortly after the patient developed blurry vision, with headaches and nausea, she displayed a loss of consciousness, with a Glasgow Coma Scale score of five (E1V1M3) and a blood pressure of 196/117 mmHg. She also exhibited bilateral fixed and dilated pupils, and brain CT showed slight high density in the cerebral base as well as diffuse brain edema involving the brain-stem, with a suspicion of subarachnoid hemorrhage and PRES (Figure 1).

MRI was not performed due to her critical condition. But we judged that it was reasonable to diagnose PRES based on risk factors, clinical manifestation and brain CT. MRI was performed on postpartum day 10 (Figure 2). FLAIR images revealed a high intense signal involving the white matter in the parietal and occipital regions.

She was confirmed to have HELLP syndrome based on the following postoperative laboratory test results: $\mathrm{AST}=131 \mathrm{IU} / 1, \mathrm{ALT}=120 \mathrm{IU} / 1, \mathrm{LDH}=922 \mathrm{IU} / 1, \mathrm{Hb}=$

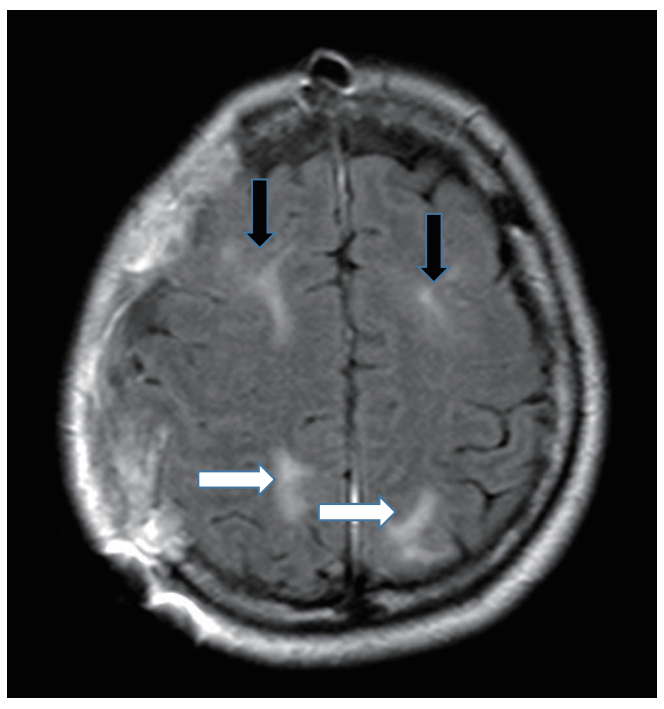

Figure 2. MRI on postpartum day 10 .

MRI, FLAIR axial image, on postpartum day 10 shows high intense signal involving white matter in parietal (black arrow) and occipital (white arrow) regions.

$14.1 \mathrm{~g} / \mathrm{dl}, \quad \mathrm{PLT}=26,000 / \mu \mathrm{l}, \quad \mathrm{Fib}=234.2 \mathrm{mg} / \mathrm{dl}$, $\mathrm{FDP}=30.0 \mu \mathrm{g} / \mathrm{ml}$, and AT-3 $=35.1 \%$. Given the considerable risk of brain herniation, as suggested by the neurosurgeon, surgical decompression for brain swelling (right fronto-temporo-parietal craniotomy) was performed. Tension of the dura matter was strong. Despite the findings of suspected subarachnoid hemorrhage on the preoperative CT assessment, no signs of hemorrhage 

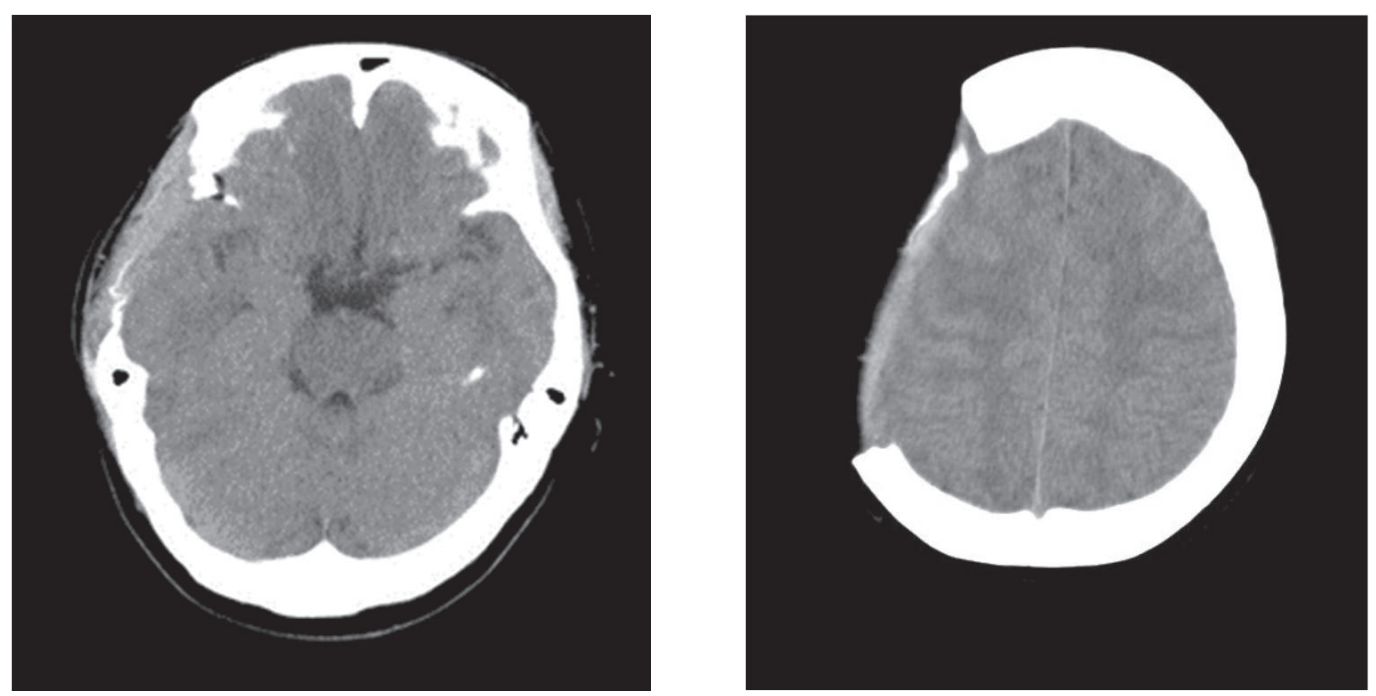

Figure 3. CT scan on postpartum day 18.

CT scan on postpartum day 18 shows significant improvement of the brain edema.

were found during surgery.

Following two days of treatment with postsurgical hypothermia in addition to intensive therapy for HELLP syndrome and DIC, she recovered her level of consciousness and was extubated on postpartum day 6 . A CT scan obtained on postpartum day 18 showed a significant improvement in the brain edema (Figure 3) and she was discharged after cranioplasty without any neurological deficits.

\section{Discussion}

PRES is a clinically radiographic syndrome first reported by Hinchey et al. in 1996.1) The clinical features of PRES include headaches, an altered mental status, seizures and visual loss ${ }^{3)}$ and neuroimaging is essential for confirming the diagnosis. Typical neurographical abnormalities include symmetrical white matter edema in the posterior cerebral hemisphere, particularly the parietooccipital region. ${ }^{4}$ Although these abnormalities are often apparent on CT scans, MRI is best for delineating the findings.

While conditions outside of pregnancy, including hypertensive encephalopathy, autoimmune diseases, hematological disorder, medications such as immunosuppressants, anticancer drugs and steroids, renal failure, liver failure and transplantation, are associated with the onset of PRES, ${ }^{5)}$ eclampsia/preeclampsia is known to cause PRES primarily in the postpartum period rather than during pregnancy. ${ }^{6}$ In this case, PRES was diagnosed on $\mathrm{CT}$ scans, not MRI, in a preeclamptic woman on the first day postpartum after emergency cesarean section for HELLP syndrome.

Although most case series have reported that PRES is fully reversible within a period of days to weeks without surgical intervention, ${ }^{7)}$ one of the largest case series highlighted the potential serious consequences of this disorder ${ }^{8)}$ In that study, which included 22 patients with PRES, the author reported six deaths and 16 patients with permanent neurological disabilities. Death may result from progressive cerebral edema, intracerebral hemorrhage or as a complication of the patient's underlying condition. Although an elevated creatinine level is associated with death, neither blood pressure levels nor its percent elevation from baseline appear to be associated with the prognosis. ${ }^{8)}$

Vasogenic edema caused by the breakdown of autoregulatory systems in brain capillaries is considered to be the main cause of PRES, ${ }^{1)}$ and is usually reversible. Cytotoxic edema, not vasogenic edema, presenting as high signals on diffusion-weighted images and low signals on ADC maps on MRI may be a predictive factor of irreversible brain ischemia. ${ }^{8)}$ In addition, more extensive brain involvement, particularly in the brainstem, correlates with a worse prognosis. ${ }^{8)}$

In the current case, although serum creatinine levels were consistently normal, the cerebral edema was severe and involved the brainstem. Consequently, we decided to perform surgical decompression in order to prevent serious neurological conditions, including death. In severe PRES, as in this case, surgical intervention should be considered as well as appropriate blood pressure management. However, it is not conclusive whether surgical intervention contributed to improvements in the patient's condition. 


\section{Acknowledgements}

This study received no foundational support.

\section{Conflicts of interest}

There are no conflicts of interest to report for any of the authors.

\section{References}

1. Hinchey J, Chaves C, Appignani B, et al. A reversible encephalopathy syndrome. N Engl J Med. 1996; 334: 494-500.

2. Fugate JE, Claassen DO, Cloft HJ, Kallmes DF, Kozak OS, Rabinstein AA. Posterior encephalopathy syndrome: associated clinical and radiologic findings. Mayo Clin Proc. 2010; 85: $427-$ 432.
3. Hefzy HM, Bartynski WS, Boardman JF, Lacomis D. Hemorhhage in posterior reversible encephalopathy syndrome: imaging and clinical features. AJNR Am J Neuroradiol. 2009; 30: 1371-1379.

4. Lamy C, Oppenheim C, Méder JF, Mas JL. Neuroimaging in posterior reversible encephalopathy syndrome. J Neuroimaging. 2004; 14: 89-96.

5. Staykov D, Schwab S. Posterior reversible encephalopathy syndrome. J Intensive Care Med. 2012; 27: 11-24.

6. Raps EC, Galetta SL, Broderick M, Atlas SW. Delayed peripartum vasculopathy: cerebral eclampsia revisited. Ann Neurol. 1993; 33: $222-225$.

7. Roth C, Ferbert A. Posterior reversible encephalopathy syndrome: long-term follow-up. J Neurol Neurosurg Psychiatry. 2010; 81: $773-777$.

8. Covarrubias DJ, Luetmer PH, Campeau NG. Posterior reversible encephalopathy syndrome: prognostic utility of quantitative diffusion-weighted MR images. AJNR Am J Neuroraiol. 2002; 23 : $1038-1048$. 